\title{
A Comparative Study of Perfusion Measurement in Brain Tumours at 3 Tesla MR: Arterial Spin Labeling versus Dynamic Susceptibility Contrast-Enhanced MRI
}

\author{
P. Lehmann ${ }^{a}$ d,g P. Monet ${ }^{a} \quad$ G. de Marco ${ }^{b} \quad$ G. Saliou ${ }^{c} \quad$ M. Perrin ${ }^{f}$ \\ S. Stoquart-Elsankari ${ }^{\text {h }} \quad$ A. Bruniaue ${ }^{\text {e J.N. Vallée }}{ }^{\text {a }}$ \\ ${ }^{a}$ Neuroradiology, Amiens University Hospital, Amiens, ${ }^{b}$ Laboratoire Contrôle Moteur et Mouvement, \\ UFR STAPS Paris X, Nanterre, 'Neuroradiology, Le Kremlin Bicetre Neuroradiology, Le Kremlin-Bicetre, \\ dLaboratoire de Neurosciences Fonctionnelles et Pathologies LFNP-UMR, and ${ }^{\mathrm{e} N o r d p a t h o l o g i e, ~ L i l l e, ~}$

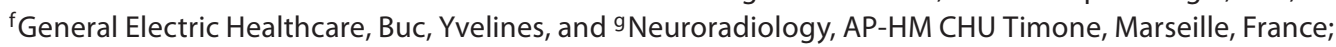 \\ h Service de Neurologie, Cliniques Universitaires Saint-Luc, Bruxelles, Belgium
}

\section{Key Words}

Arterial spin labeling • Perfusion - Cerebral blood flow .

Cerebral neoplasm · 3T MR

\begin{abstract}
Purpose: To prove the feasibility of arterial spin labeling (ASL) to explore brain tumors by comparing dynamic susceptibility contrast-enhanced MRI to ASL at 3T MR. Materials and Methods: Twenty-seven patients were included presenting 9 gliomas, 10 metastases and 8 meningiomas. All were explored by a pseudo-continuous ASL and dynamic susceptibility contrast-enhanced T2* perfusion sequence. Two neuroradiologists analyzed the cerebral blood flow (CBF) maps to assess feasibility, examination quality and quantitative comparison. The Spearman nonparametric correlation test and the BlandAltman graphic test were used to analyze our quantitative results. Results: $92 \%$ of ASL CBF maps were informative. ASL detected all lesions as well as dynamic susceptibility contrastenhanced MRI. Both sequences provide relative quantitative CBF values closely correlated. Conclusion: On a 3T MR unit, ASL is a good alternative to dynamic susceptibility contrastenhanced MRI when contrast medium is contraindicated or intravenous injection is not possible. Its results on relative CBF parameters are similar to contrast-injected perfusion.
\end{abstract}

\section{Introduction}

Perfusion MR imaging following a bolus injection of contrast agents provides useful information about vascularization. Angiogenesis is a complex process regulated by multiple stimulatory and inhibitory factors that are able to modulate the migration and/or proliferation of microvascular cells with the objective of formation of neovasculature from pre-existing vessels [1]. Angiogenesis is linked to tumor type (for example, glioblastoma is the most angiogenic tumor [2]) and angiogenesis exploration is a challenge because diagnostic and therapeutic decisions depend on tumor type [3-5]. With the development of anti-angiogenic therapy, the angiogenic process needs to be controlled regularly. Nowadays, these controls need contrast-injected perfusion imaging but contrast injection may be impossible for different reasons like renal failure, nephrogenic systemic fibrosis [6] or previous reactions. Arterial spin labeling (ASL) could be an alternative to standard intravenous bolus $\mathrm{T} 2^{*}$ perfusion. The measurement of cerebral blood flow (CBF) can be assessed with ASL, which uses magnetically labeled arterial blood water as an endogenous tracer and is based on magnetic resonance imaging (MRI). As Golay and Petersen [7] described, ASL is a very low signal-to-noise ra- 
tio (SNR) technique. Moving from 1.5 to $3 \mathrm{~T}$ allows an SNR increase but not as attempted. The T1 tissue magnetization increases; the alteration of spin-spin relaxation $\mathrm{T} 2$ and the $\mathrm{T}^{*}$ reduction limit the general SNR achievable. Another determinant factor is the main field homogeneity which is much lower at $3 \mathrm{~T}$ than at $1.5 \mathrm{~T}$. In consequence, acquisition needs the use of pulses with larger bandwidth; inhomogeneity also contributes to the worsening of artefacts in EPI images. Authors [8] had compared ASL with high to ASL with low field and proved the high-field superiority; furthermore, high-field MR units allow shorter acquisition time and a clinical use of this technique. Our purpose was to prove the feasibility of ASL to explore brain tumors by comparing standard intravenous bolus $\mathrm{T}^{*}$ perfusion with ASL perfusion.

\section{Materials and Methods}

\section{Patients}

A prospective study was conducted from October 2007 to March 2008. The local committee approved this study, patients were informed orally and consent was obtained; signed consent was not needed. The study was a consecutive series and 27 patients with cerebral tumors were included (sex ratio M/F: 19/8; mean age 58 years, min 19, $\max 82$ ): 8 cases with glioma (2 grade 2,4 glioblastoma, 1 oligodendroglioma and 1 oligoastrocytoma), 5 with meningioma and 5 with metastasis were confirmed histologically, but the other 9 were not (5 metastasis with other locations known, 3 meningioma and 1 low-grade glioma).

MR Imaging Data Acquisition

All patients were examined with a $3 \mathrm{~T}$ clinical MR imaging unit (HDX; General Electric Medical System, Milwaukee, Wisc., USA); 8-channel bird cage coil. Patients protocols are as follows:

Morphological sequences: Axial Fast SE T2, Axial SE T1, Axial FLAIR and 3D T1 after contrast injection.

Perfusion sequences:

- Before injection of contrast agent: ASL perfusion: repetition time: $9.4 \mathrm{~ms}$, echo time: $2 \mathrm{~ms}$, flip angle: $155^{\circ}$, field of view: 24 $\times 24 \mathrm{~cm}^{2}$, slice thickness: $4 \mathrm{~mm}$, NEX: 2 , number of interleaved slices: 92 , duration: 3 min $52 \mathrm{~s}$.

- During an automatic bolus injection of contrast agent (MAGNEVIST $^{\circledR}$, gadopentate dimeglumine; Bayer Sante, Puteaux, France) $(0.1 \mathrm{mmol} / \mathrm{kg})$, dynamic susceptibility contrast-enhanced MRI (DSC), axial echo-planar gradient echo imaging [9]: echo time: $30 \mathrm{~ms}$, repetition time: $1,500 \mathrm{~ms}$, flip angle: $60^{\circ}$, matrix: $128 \times 128$, field of view: $24 \times 24 \mathrm{~cm}^{2}$, slice thickness: $5 \mathrm{~mm}$, intersection gap: $1 \mathrm{~mm}$, NEX: 1 , number of slices: 20 from 65 scans. To optimize the signal-to-noise ratio and baseline, we used fat saturation on this sequence.

ASL uses the magnetization of water protons in the arterial blood stream as an endogenous, freely diffusible tracer for perfusion measurements. Any perturbation to the magnetization of the arterial blood that feeds the tissue can serve as a magnetic tracer. The perturbation is typically introduced by an RF inversion pulse at an upstream location to the tissue of interest. After a time delay that allows the magnetically labeled arterial blood to reach the tissue capillary bed, the labeled water molecules exchange with the water molecules in the tissue, causing a change in the MR signal. The spatially resolved signal change is measured by subtracting the two images acquired with labeling (label image) and without labeling (control image). The signal intensity change between label image and control image is fitted to a model from which a quantitative perfusion map of CBF is obtained. Theoretically, ASL can provide absolute values because blood properties are not affected by administration of contrast. ASL allows this determination and is not affected by blood-brain barrier rupture.

The difference image can also be viewed directly as a qualitative perfusion-weighted image.

The sequence (see 'Appendix') provided by GE Healthcare performs a pseudo continuous spin labeling for $1.5 \mathrm{~s}$ before a postspin-labeling delay of $1.5 \mathrm{~s}$. Background suppression for removing static tissue signal is optimized with saturation pulses. The image acquisition is a stack of interleaved spirals fast spin echo. The duration of each spiral is $4 \mathrm{~ms}$ readout. The host reconstruction is made using the interactive data language (IDL) code run on the scanner with the IDL virtual machine. The images are filtered with Fermi windowing to reduce ringing. Grad warp is not currently applied. After reconstruction, the images were converted into the DICOM format and inserted into the database. If at least two phases are selected, such that a reference image is available, then the difference images are converted to quantitative blood flow maps.

Data Analysis

Image processing was performed using Advantage Windows 4.4 workstation (GE Medical Systems). DSC CBF maps were analyzed by functool software (GE Medical Systems), ASL CBF maps were generated automatically. Two neuroradiologists compare the perfusion sequences (consensus). We performed:

- On ASL, an examination quality analysis using a 4-point scale and artefacts detection: examination quality: $0=$ unreadable, $1=$ poor, 2 = moderate, 3 = good.

- A qualitative comparison of the 2 sequences (ASL and standard intravenous bolus T2* perfusion) based on tumor detection: 0 = no detection, 1 = fair, 2 = good.

- A quantitative comparison (fig. 1): Based on FLAIR images to delineate peritumoral hypersignal and T1-weighted post-gadolinium images to delineate the edges of the tumor, we drew 2 regions of interest (ROI) in the tumor for each patient. We also drew a ROI on contralateral brain white matter to normalize our measures. We especially looked at cerebral blood flow $(\mathrm{CBF})$ normalized with the contralateral normal ROI: rCBF.

Statistical Analysis

Spearman's nonparametric correlation test and the Bland-Altman graphic test were used to analyze our perfusion results.

\section{Results (table 1)}

\section{ASL Perfusion Examination Quality}

92.6\% of ASL perfusion examinations were informative. Two examinations (patients 26 and 27) were unreadable $($ score $=0)$ due to dental artefacts, 1 poor $($ score $=1$ ), 10 moderate $($ score $=2)$, and $14 \operatorname{good}($ score $=3$ ). 

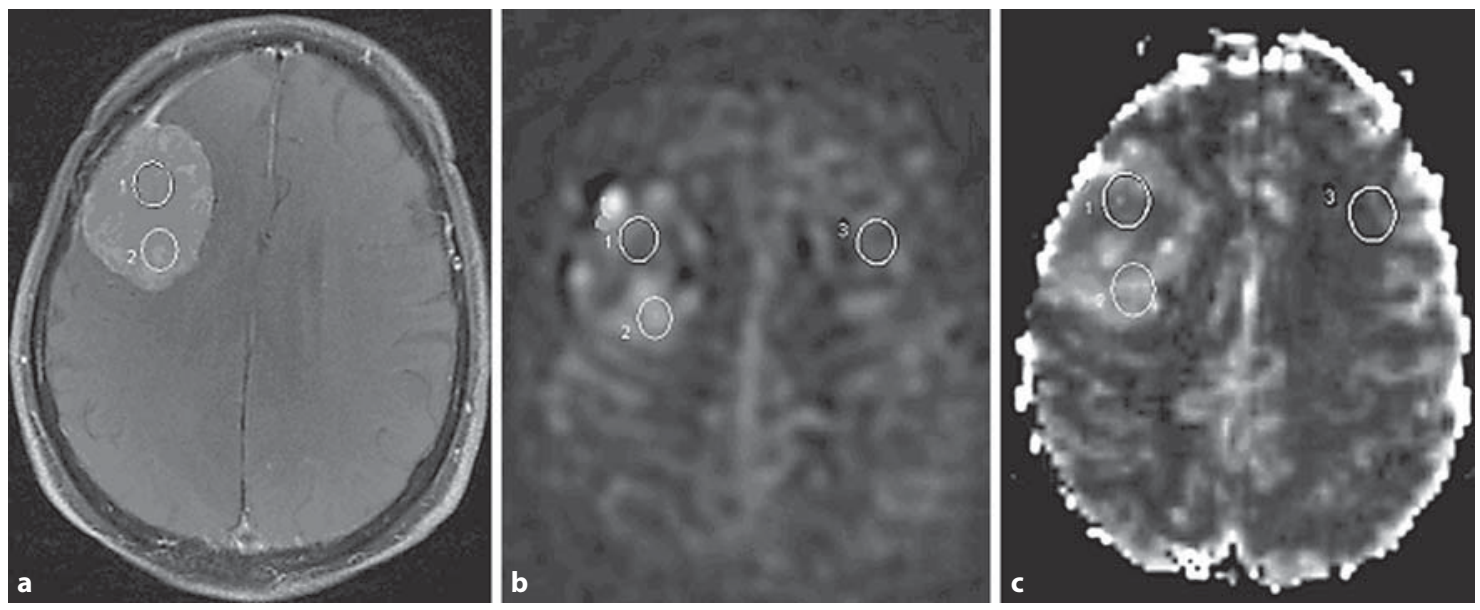

Fig. 1. ROI positioning (meningioma): 2 ROI were placed on the tumor (ROI 1 and 2), and 1 on the contralateral normal white brain matter (ROI 3). a T1 after contrast injection. b ASL CBF map. c Contrast-injected T2* perfusion CBF map.

Table 1. Qualitative and quantitative results for the 27 patients

\begin{tabular}{|c|c|c|c|c|c|c|c|c|c|c|c|}
\hline \multirow{3}{*}{$\begin{array}{l}\text { Patient } \\
\text { No. }\end{array}$} & \multirow{3}{*}{ Sex } & \multirow[t]{3}{*}{ Age } & \multirow[t]{3}{*}{ Diagnostic } & \multicolumn{2}{|c|}{ Exam quality } & \multirow{2}{*}{\multicolumn{2}{|c|}{$\frac{\text { Qualitative comparison }}{\text { tumor detection }}$}} & \multicolumn{4}{|c|}{ Quantitative comparison } \\
\hline & & & & \multirow{2}{*}{$\begin{array}{l}\text { read- } \\
\text { ability }\end{array}$} & \multirow{2}{*}{$\begin{array}{l}\text { arte- } \\
\text { facts }\end{array}$} & & & \multicolumn{2}{|c|}{ ROI 1} & \multicolumn{2}{|c|}{ ROI 2} \\
\hline & & & & & & ASL & DSC & ASL & DSC & ASL & DSC \\
\hline 1 & M & 61 & oligodendroglioma & 2 & no & 1 & 1 & 0.54 & 0.39 & 0.39 & 0.74 \\
\hline 2 & $\mathrm{~F}$ & 54 & meningioma & 3 & no & 2 & 1 & 1.77 & 0.83 & 1.84 & 0.57 \\
\hline 3 & M & 65 & metastasis & 2 & no & 1 & 1 & 1.15 & 1.42 & 0.85 & 0.77 \\
\hline 4 & $\mathrm{~F}$ & 74 & meningioma & 2 & no & 2 & 2 & 3.06 & 2.04 & 2.70 & 1.23 \\
\hline 5 & M & 68 & meningioma & 3 & no & 2 & 2 & 1.41 & 4.14 & 4.83 & 3.31 \\
\hline 6 & M & 80 & meningioma & 3 & no & 2 & 2 & 3.38 & 4.46 & 1.82 & 2.10 \\
\hline 7 & $\mathrm{~F}$ & 22 & meningioma & 3 & no & 2 & 2 & 2.51 & 2.83 & 2.86 & 2.50 \\
\hline 8 & M & 65 & metastasis & 2 & no & 2 & 2 & 0.88 & 3.60 & 3.78 & 2.88 \\
\hline 9 & $\mathrm{~F}$ & 48 & metastasis & 3 & no & 2 & 1 & 0.32 & 0.38 & 0.40 & 0.40 \\
\hline 10 & $\mathrm{~F}$ & 69 & metastasis & 3 & no & 2 & 2 & 3.88 & 3.06 & 2.11 & 2.20 \\
\hline 11 & M & 19 & oligoastrocytoma & 3 & no & 2 & 1 & 5.10 & 1.41 & 1.52 & 0.27 \\
\hline 12 & M & 52 & metastasis & 3 & no & 2 & 2 & 4.22 & 3.35 & 0.80 & 0.27 \\
\hline 13 & $\mathrm{~F}$ & 56 & metastasis & 2 & no & 2 & 2 & 0.25 & 0.05 & 0.18 & 0.04 \\
\hline 14 & M & 23 & astrocytoma grade 2 & 3 & no & 2 & 2 & 5.07 & 1.05 & 0.36 & 0.28 \\
\hline 15 & M & 60 & astrocytoma grade 2 & 3 & no & 1 & 1 & 0.65 & 0.57 & 0.45 & 0.74 \\
\hline 16 & $\mathrm{~F}$ & 82 & glioblastoma & 3 & no & 2 & 2 & 3.16 & 1.69 & 2.67 & 1.80 \\
\hline 17 & M & 62 & glioblastoma & 3 & no & 2 & 1 & 5.83 & 4.02 & 1.06 & 1.14 \\
\hline 18 & M & 78 & glioblastoma & 2 & no & 2 & 1 & 2.15 & 1.43 & 1.30 & 0.61 \\
\hline 19 & M & 40 & low-grade glioma & 3 & no & 0 & 0 & 0.64 & 0.96 & 0.65 & 0.63 \\
\hline 20 & M & 55 & glioblastoma & 2 & no & 2 & 2 & 1.25 & 0.99 & 0.68 & 0.18 \\
\hline 21 & $\mathrm{M}$ & 73 & meningioma & 2 & no & 2 & 2 & 7.59 & 3.90 & 4.34 & 1.57 \\
\hline 22 & M & 69 & meningioma & 2 & no & 1 & 1 & 1.16 & 0.53 & 1.07 & 1.92 \\
\hline 23 & $\mathrm{~F}$ & 64 & meningioma & 1 & no & 2 & 2 & 5.60 & 3.76 & 5.23 & 4.08 \\
\hline 24 & M & 63 & metastasis & 2 & no & 2 & 2 & & & & \\
\hline 25 & $\mathrm{M}$ & 57 & metastasis & 3 & no & 0 & 0 & & & & \\
\hline 26 & $\mathrm{M}$ & 59 & metastasis & 0 & yes & & & & & & \\
\hline 27 & $\mathrm{M}$ & 49 & metastasis & 0 & yes & & & & & & \\
\hline
\end{tabular}




\section{Comparison of Perfusion Sequences}

Qualitative Comparison for Tumor Detection

ASL perfusion examinations were compared to DSC perfusion; the 2 examinations that were unreadable on ASL perfusion were not compared. In the 25 patients compared, all lesions but one (patient 25) were detected by the 2 sequences. Therefore, 5 lesions were detected with a good result (score $=2$ ) by ASL perfusion and with a fair result (score $=1$ ) by DSC perfusion. A 9-mm lesion (patient 25) was not detected by ASL or by DSC perfusion.

\section{Quantitative Comparison}

Twenty-three examinations were compared; 4 patients were not because of hemorrhagic lesions (patient 24), too small lesions $(9 \mathrm{~mm})$, not being visible on both the perfusion sequences (patient 25), or poor examination quality (patients 26 and 27).

Based on the results displayed in table 1, Spearman's nonparametric correlation test was for ROI $1: \mathrm{r}=0.69$, $\mathrm{p}<0.001$ and for ROI 2: $\mathrm{r}=0.784, \mathrm{p}<0.0001$. These results showed a significant correlation between $\mathrm{rCBF}$ calculated from the 2 perfusion sequences. The Bland-Altman test confirmed the results for the ROI.

\section{Discussion}

ASL provides perfusion that is comparable to contrastenhanced MR perfusion. There are several ASL techniques, which are commonly classified as continuous (CASL $[10,11])$ or pulsed ASL (PASL $[12,13])$. CASL produces the largest ASL signal change, potentially the greatest SNR, and can be advantageous. PASL is interesting because of its straightforward implementation and the high and stable tagging efficiency with respect to flow velocity. In contrast, CASL benefits from the longer and better temporally defined tagging bolus and is consequently higher than in PASL. The popularity of CASL, however, has been mainly hindered by the requirement of a continuous-mode RF transmission, which is not always available on commercial imagers. Furthermore, the sensitivity of adiabatic inversion to flow velocity leads to a tagging efficiency that is mostly around $90 \%$ and can be as low as $68 \%$ when the amplitude-modulating method is used [14]. More recently, a train of discrete RF pulses was proposed to generate the tag and control states in ASL. This method, termed pseudocontinuous ASL (pCASL), has the potential of combining the advantages of PASL and CASL. It includes a longer tagging bolus and thus higher SNR, therefore providing a better balance between tagging efficiency and SNR [14].

Here we propose to use the method described previously $[15,16]$ for flow-driven adiabatic inversion that employs repeated RF pulses rather than continuous RF. This method potentially overcomes a number of the limitations of previous implementations of CASL. Furthermore, this sequence, provided by GE Healthcare, is a 3D sequence.

Based on our results, ASL allows a three-dimensional whole exploration of brain tumors without an injection of contrast agents. Moreover, CBF maps are automatically created without human postprocessing and its short acquisition time ( 3 min $52 \mathrm{~s}$ ) on a 3T MR unit allows a routinely clinical use. Previous studies on a 1.5T MR unit [17-19] already show that ASL is a suitable method for the assessment of microvascular perfusion but the acquisition time was higher and clinical use more difficult. Furthermore, compared to $1.5 \mathrm{~T}$ previous studies, our study proves that a 3T MR unit can be used for ASL imaging without an increase of artefacts.

Our results show that there is a close correlation between ASL and standard intravenous bolus T2* perfusion in the determination of $\mathrm{rCBF}$ in cerebral tumors. Most of the maps obtained (92.6\%) were interpretable, and the 2 unreadable ones were linked to dental artefacts which also disturb the interpretation of DSC perfusion. The comparison we made shows good results in a qualitative and quantitative manner with a good correlation. The ASL provides similar information as DSC perfusion for rCBF measurement. Other ASL sequences had already been compared to DSC. Even if Ludemann et al. [20] conclude that tumor perfusion values determined with a different technique are not comparable, studies on gliomas $[17,21]$ or meningiomas [18] found a good correlation between ASL and DSC for determination of CBF. We obtain the same conclusion with this pCASL sequence, and a recent study led to the same conclusions [22]. Like DSC perfusion, ASL can guide cerebral biopsy to the most angiogenic part of the tumor [23-25]. Some authors [26] propose to associate ASL and diffusion-weighted imaging for permeability measurement.

ASL presents limits. First of all, ASL only provides $\mathrm{CBF}$ quantification. But even if cerebral blood volume (CBV) obtained with DSC perfusion is the parameter used most often, several studies report the same diagnostic value of $\mathrm{CBF}[17,27,28]$.

$\mathrm{CBF}$ quantification may be affected by transit time which differs from gray to white matter $[29,30]$ and may vary with patient age [31]. Therefore, CBF may be underestimated with ASL at low flow rates. 
The entire posterior fossa exploration is not possible with this sequence because of the inversion labeling which makes the first centimeters unavailable. This limit must be considered for posterior fossa tumors even if DSC perfusion often presents image distortion caused by susceptibility artefacts, chemical shift artefacts, or ghosts in the same region.

Finally, continuous labeling requires near-continuous wave RF transmit capability that is often not available on imagers. Multislice implementations of CASL [32, 33] suffer from signal attenuation, velocity sensitivity or imperfect static tissue subtraction or require special hardware and labeling geometries.

ALS presents majors advantages: first, for the patient, the fact that no contrast is used which is an advantage in patients who might develop nephrogenic systemic fibrosis, and, second, ASL is less susceptible to the effects of the blood barrier breakdown than contrast-enhanced MR perfusion.

\section{Conclusion}

ASL perfusion is a good alternative to DSC perfusion when an injection of contrast agent is not possible. A 3T MR unit can be used for ASL imaging without an increase in artefacts and with an acceptable time for clinical use. This preliminary study on CBF parameters shows similar results to those obtained with conventional perfusion studies following an injection of contrast medium. Its role could also be considered for cerebral tumor follow-up.

\section{Conflict of Interest}

Muriel Perrin is employed by General Electric Healthcare as an engineer.

\section{Appendix}

\section{D Spiral ASL Sequence and Quantification (12/10/07 Version)}

The prototype 3D ASL sequence is a combination of a background-suppressed CASL preparation, a 3D stack of spiral fse readout, and a host recon that reconstructs quantitative images. The three parts will be described in order.

\section{ASL Preparation}

Continuous labeling is performed for $1.5 \mathrm{~s}$ prior to a postlabeling delay that defaults to $1.5 \mathrm{~s}$. Continuous labeling is achieved with the pulsed continuous approach introduced at the ISMRM in 2005 [16] and 2008 [15]. Our in vivo measurements indicate that the absolute efficiency of this pulsed continuous approach is $81 \%$ for the parameters chosen here. Background suppression is achieved with a number of pulses (see figure below). Selective spatial saturation and inversion are applied prior to labeling and then minimally selected inversions are applied after labeling to maintain background suppression. Inferior saturation pulses are applied at times corresponding to the background suppression nulls of blood to minimize artefacts from inflowing blood.

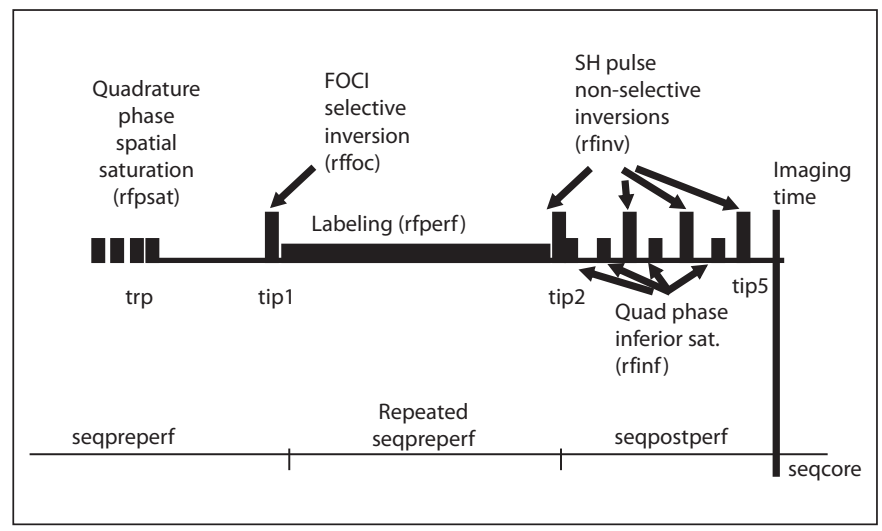

Image Acquisition

The image acquisition is a stack of spirals fse. The number of slice echoes are acquired with centric ordering after each preparation. The spirals are interleaved and different interleaves are acquired following different preparations.

Quantification is performed as follows:

$$
C B F=6,000 \frac{\lambda\left(1-\exp \left(-\frac{2.0 s}{1.2 s}\right)\right) \exp \left(\frac{w(s)}{T 1}\right)}{2 T 1(s)\left(1-\exp \left(-\frac{1.5}{T 1(s)}\right)\right) \varepsilon}\left(\frac{\text { ASLdiff }}{(45.25 \text { nex }) \text { PDref }}\right)
$$

where $\mathrm{T} 1$ of $1.6 \mathrm{~s}$ for blood at $3 \mathrm{~T}$ or $1.4 \mathrm{~s}$ for blood at $1.5 \mathrm{~T}$ is assumed. The partial saturation of the 'PD' reference image is corrected for a T1 of $1.2 \mathrm{~s}$ typical of gray matter. While a more fully relaxed signal would be desirable, the saturation of the receiver and the bright signal on the slightly T2-weighted fse makes this undesirable. The partition coefficient, $\lambda$, was set to the whole brain average, 0.9 , and the efficiency, $\varepsilon$, is set to $0.95 \times 0.75$ to account for the combined efficiency of labeling and suppression. Our more recent numbers suggest this should be $0.8 \times 0.75$ so flow is underestimated by $15 \%$. This quantification assumes the label remains in blood and thus $\mathrm{T} 1$ of the tissue, and the delay in the arrival time to the tissue, need not be quantified. 


\section{References}

1 Folkerth RD: Descriptive analysis and quantification of angiogenesis in human brain tumors. J Neuro-oncol 2000;50:165-172.

$\checkmark 2$ Kleihues P, Louis DN, Scheithauer BW, et al: The WHO classification of tumors of the nervous system. J Neuropathol Exp Neurol 2002;61:215-225; discussion 226-219.

- 3 Giese A, Westphal M: Treatment of malignant glioma: a problem beyond the margins of resection. J Cancer Res Clin Oncol 2001; 127:217-225.

4 Law M, Young RJ, Babb JS, et al: Gliomas: predicting time to progression or survival with cerebral blood volume measurements at dynamic susceptibility-weighted contrastenhanced perfusion MR imaging. Radiology 2008;247:490-498.

5 Schiff D: Single brain metastasis. Curr Treat Options Neurol 2001;3:89-99.

6 Wiginton CD, Kelly B, Oto A, et al: Gadolinium-based contrast exposure, nephrogenic systemic fibrosis, and gadolinium detection in tissue. AJR Am J Roentgenol 2008;190: 1060-1068.

7 Golay X, Petersen ET: Arterial spin labeling: benefits and pitfalls of high magnetic field. Neuroimaging Clin N Am 2006;16:259-268.

-8 Wang J, Alsop DC, Li L, et al: Comparison of quantitative perfusion imaging using arterial spin labeling at 1.5 and 4.0 Tesla. Magn Reson Med 2002;48:242-254.

-9 Knopp EA, Cha S, Johnson G, et al: Glial neoplasms: dynamic contrast-enhanced $\mathrm{T}_{2}{ }^{*}$ weighted MR imaging. Radiology 1999;211: 791-798.

10 Detre JA, Leigh JS, Williams DS, Koretsky AP: Perfusion imaging. Magn Reson Med 1992;23:37-45.

$\checkmark 11$ Williams DS, Detre JA, Leigh JS, Koretsky AP: Magnetic resonance imaging of perfusion using spin inversion of arterial water. Proc Natl Acad Sci USA 1992;89:212-216.

$\checkmark 12$ Edelman RR, Siewert B, Darby DG, et al: Qualitative mapping of cerebral blood flow and functional localization with echo-planar MR imaging and signal targeting with alternating radio frequency. Radiology 1994; 192:513-520.
13 Kim SG: Quantification of relative cerebral blood flow change by flow-sensitive alternating inversion recovery (FAIR) technique: application to functional mapping. Magn Reson Med 1995;34:293-301.

14 Wu WC, Fernandez-Seara M, Detre JA, Wehrli FW, Wang J: A theoretical and experimental investigation of the tagging efficiency of pseudocontinuous arterial spin labeling. Magn Reson Med 2007;58:1020-1027.

15 Dai W, Garcia D, de Bazelaire C, Alsop DC: Continuous flow-driven inversion for arterial spin labeling using pulsed radio frequency and gradient fields. Magn Reson Med 2008;60:1488-1497.

16 Garcia DM, De Bazelaire C, Alsop DC: Pseudo-continuous Flow Driven Adiabatic Inversion for Arterial Spin Labeling. Miami, ISMRM, 2005

17 Warmuth C, Gunther M, Zimmer C: Quantification of blood flow in brain tumors: comparison of arterial spin labeling and dynamic susceptibility-weighted contrast-enhanced MR imaging. Radiology 2003;228: 523-532.

18 Kimura H, Takeuchi H, Koshimoto Y, et al: Perfusion imaging of meningioma by using continuous arterial spin-labeling: comparison with dynamic susceptibility-weighted contrast-enhanced MR images and histopathologic features. AJNR Am J Neuroradiol 2006;27:85-93.

19 Weber MA, Zoubaa S, Schlieter M, et al: Diagnostic performance of spectroscopic and perfusion MRI for distinction of brain tumors. Neurology 2006;66:1899-1906.

20 Ludemann L, Warmuth C, Plotkin M, et al: Brain tumor perfusion: comparison of dynamic contrast enhanced magnetic resonance imaging using $\mathrm{T} 1, \mathrm{~T} 2$, and $\mathrm{T} 2 *$ contrast, pulsed arterial spin labeling, and $\mathrm{H} 2(15) \mathrm{O}$ positron emission tomography. Eur J Radiol 2009;70:465-474.

21 Weber MA, Gunther M, Lichy MP, et al: Comparison of arterial spin-labeling techniques and dynamic susceptibility-weighted contrast-enhanced MRI in perfusion imaging of normal brain tissue. Invest Radiol 2003;38:712-718.

-22 Jarnum H, Steffensen EG, Knutsson L, et al: Perfusion MRI of brain tumours: a comparative study of pseudo-continuous arterial spin labelling and dynamic susceptibility contrast imaging. Neuroradiology 2010;52:307317.
23 Kim MJ, Kim HS, Kim JH, Cho KG, Kim SY: Diagnostic accuracy and interobserver variability of pulsed arterial spin labeling for glioma grading. Acta Radiol 2008;49:450-457.

$>24$ Tourdias T, Rodrigo S, Oppenheim C, et al: Pulsed arterial spin labeling applications in brain tumors: practical review. J Neuroradiol 2008;35:79-89.

25 Wolf RL, Wang J, Wang S, et al: Grading of CNS neoplasms using continuous arterial spin labeled perfusion MR imaging at 3 Tesla. J Magn Reson Imaging 2005;22:475-482.

26 Wang J, Fernandez-Seara MA, Wang S, St Lawrence KS: When perfusion meets diffusion: in vivo measurement of water permeability in human brain. J Cereb Blood Flow Metab 2007;27:839-849.

27 Petersen ET, Lim T, Golay X: Model-free arterial spin labeling quantification approach for perfusion MRI. Magn Reson Med 2006; 55:219-232.

28 Wang J, Alsop DC, Song HK, et al: Arterial transit time imaging with flow encoding arterial spin tagging (FEAST). Magn Reson Med 2003;50:599-607.

29 Gunther M, Bock M, Schad LR: Arterial spin labeling in combination with a look-locker sampling strategy: inflow turbo-sampling EPI-FAIR (ITS-FAIR). Magn Reson Med 2001;46:974-984

30 Ye FQ, Berman KF, Ellmore T, et al: H(2)(15) $O$ PET validation of steady-state arterial spin tagging cerebral blood flow measurements in humans. Magn Reson Med 2000;44:450456.

-31 Parkes LM, Rashid W, Chard DT, Tofts PS: Normal cerebral perfusion measurements using arterial spin labeling: reproducibility, stability, and age and gender effects. Magn Reson Med 2004;51:736-743.

32 Alsop DC, Detre JA: Multisection cerebral blood flow MR imaging with continuous arterial spin labeling. Radiology 1998;208: 410-416.

33 Silva AC, Zhang W, Williams DS, Koretsky AP: Multi-slice MRI of rat brain perfusion during amphetamine stimulation using arterial spin labeling. Magn Reson Med 1995; 33:209-214. 